\title{
ANÁLISIS DE LA CULTURA PREVENTIVA FRENTE A LOS INCENDIOS URBANOS EN LOS COMERCIANTES DEL MERCADO ANEXO I DE PIURA
}

\author{
Mirian Yanet Velasco Pasapera, Jimena Moscol Seminario, Lady Diana Celi Zapata y Sheyla Alexandra Cornejo Medina \\ Universidad César Vallejo - Perú \\ Recibido: 01/06/2020. Aprobado: 26/10/2020 \\ Cómo citar este artículo: \\ Velasco Pasapera, M.Y., Moscol Seminario, J., Celi Zapata, L.D. y Cornejo Medina, A. (2020). Análisis de la cultura preven- \\ tiva frente a los incendios urbanos en los comerciantes del Mercado Anexo I de Piura. Revista Sapientía, 12 (24), 36-45.
}

\section{RESUMEN}

La presente investigación tuvo como objetivo general analizar la cultura preventiva frente a los incendios urbanos de los comerciantes del Mercado Anexo I de la ciudad de Piura, esto con base en la teoría de los cinco niveles de cultura preventiva, propuesta por Parker (2016). El diseño de la investigación fue cuantitativo y la muestra fue de 20 responsables de las tiendas y puestos de trabajo del recinto. La técnica usada fue la entrevista a través de cuestionario, concluyendo que existe una carencia en relación a cultura preventiva, evidenciándose, por un lado, las estadísticas del cuerpo de bomberos de la ciudad de Piura y, por otro lado, los resultados tras aplicada la encuesta. Así mismo, los comerciantes antes del incendio se situaban en un nivel de cultura preventiva patológico; después del siniestro se ubican en el nivel reactivo.

\section{PALABRAS CLAVE}

Mercado, cultura, prevención, patológico, formalista, reactivo, proactivo y generalista.

\section{ABSTRACT}

The main objective of this research was to analyze the preventive culture against urban fires in Annex I Market traders in Piura city, based on the theory of the five levels of preventive culture, proposed by Parker (2016). The design of the research was quantitative and was made up of 20 managers of the enclosure. The technique used was the interview through questionnaire. Concluding that, there is a lack of in relation to preventive culture; showing, on the one hand, which the statistics rovided by the fire brigade of Piura city and, on the other hand, the results after the survey were applied. After the sinister, they are located at the Reactive level.

\section{KEYWORDS}

Market, culture, preventive, pathological, reactive, formalist, proactive and generalist. 


\section{INTRODUCCIÓN}

En el mundo los incendios han aumentado considerablemente, más aún en la ciudad de Piura, en donde el cuerpo de Bomberos Piura $\mathrm{N}^{\circ}$ 25 ha reportado 29 siniestros hasta febrero del 2020 y 193 en el año 2019. Haciendo una revisión histórico temporal, el Compendio Estadístico Piura INDECI (2017) señaló que en 2010 se reportaron 2387 emergencias; en 2013, 751 emergencias y en 2012 ocurrieron 464 emergencias. En este último, se destacó el incendio urbano en el A.A H.H Los Vencedores (Sullana) dejando un total de 35 personas damnificadas, 294 afectadas, 10 viviendas colapsadas, 80 animales perdidos y 80 personas afectadas por el servicio del agua (INDECI, 2013). En el año 2014, el número de incendios llegó a 704; mientras que en el 2015 a 692, y en el año 2016 a 756. Por otro lado, en el año 2016 el Mercado de Piura se vio afectado en un $70 \%$ por un incendio, causando un aproximado de 50 millones de soles en pérdidas. Al respecto, Andina (2016) indicó que se habría originado por un cortocircuito debido a las conexiones; sin embargo, el Diario Correo (2016) manifestó que se produjo por una chispa de soldadura. Luego de este incidente, en el 2018, Gina Bustinza López encabezó una inspección al Mercado Anexo, la cual reveló que este centro de abastos no tiene extintores suficientes para atender una emergencia; algunas vías de acceso al mercado están obstaculizadas por algunos productos de los mismos comerciantes y cuenta con la señalización respectiva (Cutivalú, 2018). La problemática está latente e INDECI (2018) resalta que las causas de estos siniestros obedecen a fallas en las instalaciones eléctricas, fugas de gas, manejo inadecuado de materiales inflamables, entre otros.

Considerando esta problemática, surge la necesidad investigar respecto a los incendios urbanos, en tal sentido se plantea el siguiente interrogante: $i L o s$ comerciantes de las tiendas y puestos de trabajo del Mercado ANEXO I de Piura tienen cultura de prevención frente a incendios urbanos?
Entre las investigaciones internacionales está el trabajo de Vázquez et al. (2017), que tuvo como objetivo incrementar los conocimientos sobre reducción del riesgo de desastres en la población perteneciente a los Consultorios Médicos 5 y 12, y llegó a la conclusión de que la población tiene baja percepción del riesgo, lo que llevó a elaborar una estrategia educativa comunitaria. El trabajo de Ordóñez (2015) tuvo como objetivo realizar un estudio sobre el grado de cumplimiento de las normas de seguridad de los sistemas de prevención contra incendio en los locales de las Pymes (Guayaquil), concluyendo que apenas el $40 \%$ de los establecimientos reúnen todas las normas mínimas requeridas para la prevención de incendio. Por su parte, Rastelli (2013) orientó su investigación a determinar las estrategias para integrar la reducción del riesgo en la gestión municipal de Chacao como elemento de la sostenibilidad; su trabajo llegó a dos conclusiones importantes: primero, que la alcaldía desconoce qué parte de sus funciones es la reducción del riesgo y, segundo, la necesidad de integrar la gestión del riesgo en la planificación de la gestión municipal.

Alvarado (2013) en su investigación se propuso determinar la evaluación de la gestión de riesgos a los desastres en los procesos administrativos en la educación universitaria superior; sus conclusiones indican que cerca del 60\% ignoraba la existencia de un Plan de Emergencia, mientras el $64.6 \%$ nunca se había capacitado en gestión del riesgo; un 56.1\% conocía sobre primeros auxilios y un $64.6 \%$ ignora cómo usar un extintor. Entretanto, Hernández (2011) analizó el conocimiento y formación en cultura preventiva de riesgo en los Centros de Enseñanza; sus conclusiones revelaron que la seguridad en las escuelas sigue sin consolidarse, además no existe mucho interés por transmitir un mensaje auto protector, lo que pone en evidencia una baja cultura de prevención en sus docentes por su falta de compromiso al respecto.

En Perú, Neuhaus (2013) tuvo como objetivo identificar factores que limitan una implementación 
efectiva dela gestión del riesgo de desastres, lo quellevó a concluir qué zonas de mayor exposición presentaron algún compromiso con la gestión reactiva, pero también puso en evidencia la incertidumbre sobre cómo implementar el SINAGERD.

Esta investigación se justifica teóricamente con la información obtenida desde el año 1999 sobre cultura de prevención. En este sentido, será posible conocer en qué medida ayuda a las personas a identificar, reducir, prevenir, prepararse, reaccionar y recuperarse ante un incendio. Asimismo, se justifica metodológicamente puesto que es un trabajo que permitirá estructurar preguntas que permitan ampliar perspectivas frente a incendios urbanos de las tiendas y puestos de trabajo del Mercado Anexo I de la ciudad de Piura. Por último, se justifica de manera práctica debido a que, una vez analizada la información recopilada, permitirá en posteriores investigaciones realizar programas de prevención frente a los incendios urbanos con el fin de disminuir el número de casos ocurridos.

La presente investigación tiene por objetivo general analizar la cultura preventiva frente a incendios urbanos de los comerciantes del mercado ANEXO I de Piura, cuyos objetivos específicos son conocer la dimensión patológica, la dimensión reactiva, la dimensión formalista, la dimensión proactiva y la dimensión generativa de la variable cultura preventiva en los comerciantes del mercado Anexo I de Piura.

\section{MARCO TEÓRICO}

Un incendio es un fuego de grandes proporciones (Junta de Andalucía, s/n), que puede presentarse de manera instantánea o gradual, provocando daños materiales, pérdida de vidas humanas y afectación al ambiente (INDECI, 2018). La Norma Técnica Peruana, NTP (2012), clasifica el fuego en 5 categorías: CLASE A, son todos los incendios provocados por materiales orgánicos sólidos como el papel, madera, cartón, tela y algunos tipos de plástico; CLASE $\mathrm{B}$, son todos los fuegos alimentados por líquidos inflamables y materiales que arden fácilmente, por ejemplo: gasolina, diésel, bunker, parafina, cera, plásticos, alquitrán y alcoholes; CLASE C, incendios alimentados por equipos eléctricos energizados. Por ejemplo: computadoras, servidores, maquinaria industrial, herramientas eléctricas, hornos eléctricos y microondas etc.; CLASE D, fuegos alimentados por ciertos tipos de metales, como el sodio, potasio, polvo de aluminio, básicamente metales alcalinos y alcalinotérreos; CLASE K, fuego provocado por aceite de cocina, específicamente en freidoras (aceite vegetal, animal, grasa, etc.), debido a que el aceite de cocina es muy difícil de apagar y que reacciona violentamente al contacto con agua, se usa específicamente el extintor de clase K. Esta clasificación resulta de vital importancia para saber qué tipo de extintor usar en caso de incendio.

Sin embargo, una vez iniciado el incendio se puede clasificar según su localización en: incendios industriales, suceden en industrias o instalaciones donde se almacenan o fabrican sustancias combustibles; incendios forestales: el combustible es sólido ardiendo la masa forestal, en donde hay tres subtipos: de copas (el fuego se extiende en las copas de los árboles), de superficie (arden matorrales, herbáceas y hojas secas) y de subsuelo (arden las raíces de los árboles y otra materia orgánica); incendios de transportación suceden en vehículos y medios de transporte de personas o productos; e incendios urbanos: suceden en lugares donde existe concentración humana como casas o edificios (Ruva Incendios, s/f). Al respecto, INDECI (2010) menciona que usualmente este tipo de incendio es causado por fallas en las instalaciones eléctricas, fugas de gas, manejo inadecuado de materiales inflamables, velas encendidas, mantenimiento deficiente de tanques contenedores de gas, entre otras; en tanto las causas son variadas, es preciso que la ciudadanía tenga cultura de prevención.

El concepto de "cultura de prevención" o "cultura preventiva" nace en los años 80 a consecuencia del accidente de Chernóbil. En un principio sólo hacía referencia a grandes accidentes, pero con el paso del tiempo engloba el conjunto de riesgos, implicando a las empresas en su totalidad, desde el director general 
de la empresa hasta los trabajadores (ENVIRA, 2018). Actualmente se entiende como el conjunto de actitudes y creencias positivas (compartidas por todos en la empresa) sobre salud, riesgos, accidentes, enfermedades y medidas preventivas (Prevencionar, 2017). En otras palabras, es la preocupación que tiene una sociedad por el bienestar de sus ciudadanos.

Según Parker (2016), existen cinco niveles de cultura preventiva en los que se puede clasificar las organizaciones; estos niveles son: patológico, en este nivel se encuentran las empresas que únicamente actúan en caso de sanción o imposición por parte de la autoridad; nivel reactivo, en este nivel se encuentran las que actúan cuando el accidente ya se ha producido; nivel formalista: en este nivel se disponen de un sistema de gestión en el que se generan datos de seguridad y salud, se ejecutan auditorías de seguridad y salud y se modifican procesos; nivel proactivo, en este nivel, como la propia palabra indica, se encuentran las empresas en las que se realizan mejoras continuamente, toda la empresa está implicada y comprometida, se produce un trato bidireccional lo cual genera más confianza y la realización de auditorías cada vez es menos necesaria, y el nivel generativo, en este nivel las empresas se adelantan a los errores antes de que ocurran y estos son usados para mejorar, por lo que la seguridad está implícita en todas las decisiones y por encima de todo (ENVIRA, 2018).

Así mismo, la cultura preventiva cuenta con dos elementos básicos: dirección y educación preventiva. En la dirección se engloba a todos aquellos actores que tienen un rol de mando dentro de una organización pública o privada (directores, gerentes, mandos intermedios directivos $y$ presidentes), estos actores son los primeros que deben estar concientizados y deben dar un ejemplo continuo para crear y fomentar dicha cultura dentro de la organización, ya sea pública o privada, deben participar en todas las actuaciones que tengan que ver con la seguridad y salud en su organización; deben fomentar la implantación, premiar la implicación y la creatividad en la aplicación o desarrollo de dichas medidas (Prevencionar, 2017).
Por su parte, educación involucra que las personas consigan instaurar en sus vidas este tipo de cultura, creando conciencia de que un problema puede ser evitable en muchos casos, adoptando una actitud responsable y de respeto por la vida.

En este orden de ideas, se indica que para lograr una correcta cultura de prevención debemos empezar desde abajo; es decir, creando una educación preventiva, ya que de nada sirve que se tengan normas, procedimientos y buenos equipos si llega a fallar el factor humano, en donde se destacan habilidades tales como liderazgo, confianza y apoyo (ENVIRA, 2018). Queda claro que, el imponer aquella educación resulta difícil, pero ha de empezarse desde cero; se trata de enriquecer las creencias y actitudes positivas compartidas por todas las personas de manera que se promuevan mejores condiciones de salud y de trabajo (ENVIRA, 2018). Todo este proceso requiere una participación colectiva de toda la sociedad en su conjunto; por tanto, conseguir la implantación de una cultura preventiva efectiva implica un largo proceso social donde las personas y las estructuras organizacionales deben contribuir a su desarrollo. Esto debe iniciar desde las inspecciones constantes, debido a que, tal como lo menciona Del Prado (2019), ayudarán a descubrir si cada establecimiento cuenta con algún equipo desgastado y/o ya han llegado a su límite de condición; así mismo detectar si su capacidad es deficiente $o$ se ha usado inadecuadamente, ayudando a detectar los métodos y las prácticas que poseen potencialidad de daño.

\section{METOdOLOGÍA}

\section{Diseño de investigación}

El diseño de la investigación fue cuantitativo, debido a que dentro de su visión permite obtener resultados concretos y fiables que respondan a los objetivos de investigación con base de medición numérica y análisis estadísticos (Del Canto y Silva, 2013).

\section{Procedimiento}

El procedimiento para la recolección de información fue: indagar el número poblacional de estudio del 
Mercado Anexo I, el cual, según la Municipalidad Provincial de Piura (2011), cuenta con aproximadamente 604 tiendas y puestos de trabajo, para lo cual la muestra de estudio estuvo conformada por un total de 20 responsables de tiendas y puestos de trabajo. Seguidamente, se seleccionó el número de tiendas para entrevistar a los encargados (siendo el jueves 20 y sábado 22 de febrero 2020 por la mañana); luego, se describió de manera precisa el objetivo principal de la investigación y se les brindó la libertad de elegir participar o no del mismo. A continuación, las investigadoras elaboraron las preguntas del cuestionario correspondientes a cultura de prevención frente a incendios urbanos $y$, para facilitar la respuesta de los participantes, se aclaró las instrucciones; finalmente se agradeció por la participación.

\section{Aspectos éticos}

El procedimiento de recolección de datos de esta investigación no atentará contra la seguridad de los entrevistados; los resultados de la investigación serán realizados de modo transparente y abierto para las futuras investigaciones.

\section{RESULTADOS}

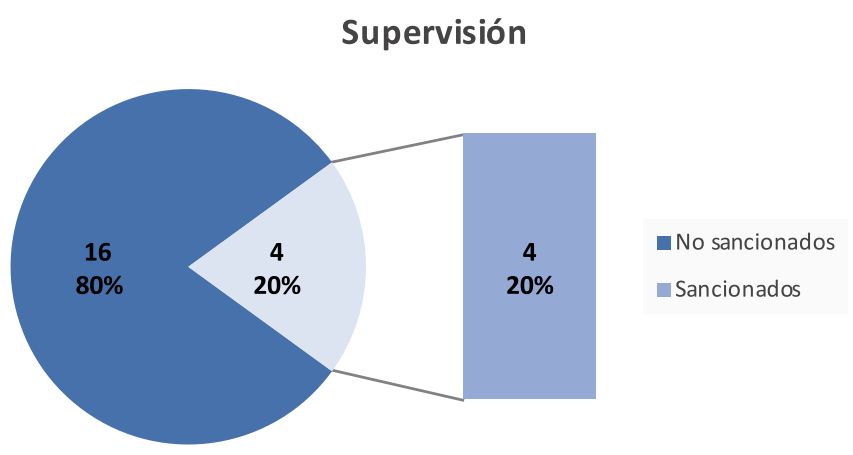

Gráfico 1: Dimensión patológica de la cultura preventiva en los comerciantes del mercado Anexo I Piura

Respecto a la dimensión patológica se pudo encontrar que 17 de los 20 evaluados manifiestan que nunca han sido supervisados por Defensa Civil y ACOMIN. Por otro lado, se conoció que 16 de los 20 comerciantes no han sido sancionados por las mismas entidades, continuando en el estado en el que se encuentran; así mismo; mientras los 4 restantes sí han sido sancionados, de los cuales 3 sí ha levantado las observaciones que se le hicieron y sólo 1 se mostró indiferente.

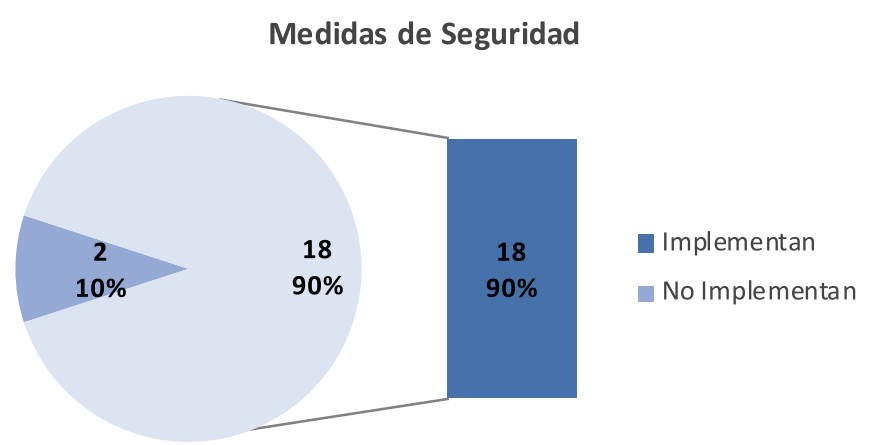

Gráfico 2: Dimensión Reactiva de la cultura preventiva en los comerciantes del Mercado Anexo I Piura

En cuanto a la dimensión reactiva, se puede encontrar que 18 de los 20 comerciantes se han preocupado por implementar medidas de seguridad contra incendios tras ocurrido el siniestro en el año 2016, tales como contar con 2 extintores, uno de $10 \mathrm{~kg}$ y uno de $20 \mathrm{~kg}$ por puesto; mientras que los otros 2 se mostraron indiferentes ante el suceso no tomando medidas preventivas. Por otro lado, solo 2 puestos, uno de golosina y otro de confecciones, instalaron detectores de humo.

\section{Establecimientos en Regla}

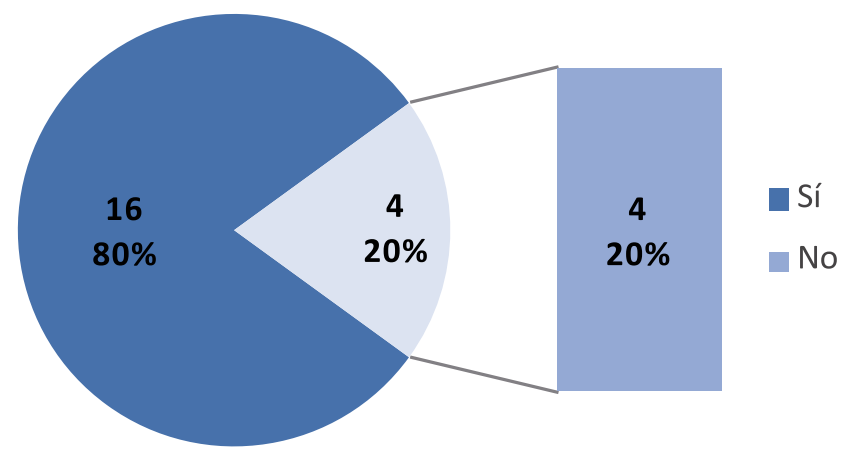

Gráfico 3: Dimensión Formalista de la cultura preventiva en los comerciantes del Mercado Anexo I Piura 
En la dimensión formalista se encontró que 16 de los 20 comerciantes han levantado observaciones después de haber sido supervisados por ACOMIN y/o Defensa Civil luego de ocurrido el incendio. Los 4 evaluados restantes no han sido supervisados; sin embargo, 2 de ellos procuran mantener todo en orden, es decir, tienen a la vista su extintor con su respectiva señalización, botiquín, llave térmica de luz en buen estado; mientras los otros 2 omiten o les resulta indiferente pese al incendio ya ocurrido.

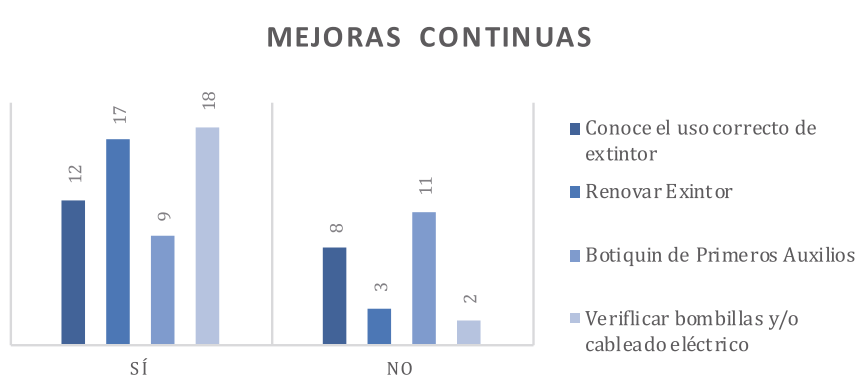

Gráfico 4: Dimensión Proactiva de la cultura preventiva en los comerciantes del Mercado Anexo I Piura

En la dimensión proactiva se encontró que 17 de 20 evaluados cambian de los extintores que tienen dentro de su puesto cada año, dado que los distribuidores de extintores llegan a cada puesto para informarles la fecha en que deben realizar el cambio. Los 3 puestos restantes son indiferentes ante esos cuidados. Por otro lado, 11 de 20 responsables de los puestos no renuevan su botiquín de emergencias. De igual modo, sólo 18 de 20 responsables verifican bombillas y/o cableado eléctrico de su puesto de trabajo de forma continua; mientras los otros 2 restantes no realizan dichas verificaciones debido a que el costo que conlleva realizarlo sería mayor a los ingresos que tienen. Finalmente, 12 de 20 responsables de los puestos refieren mantener al personal capacitado en el uso de extintores, sin embargo, al preguntarles directamente cómo lo utilizarían, manifiestan no saber emplearlo; de la misma forma, los responsables de los 8 puestos restantes afirman haber recibido charlas, pero en la práctica tampoco saben usarlo.

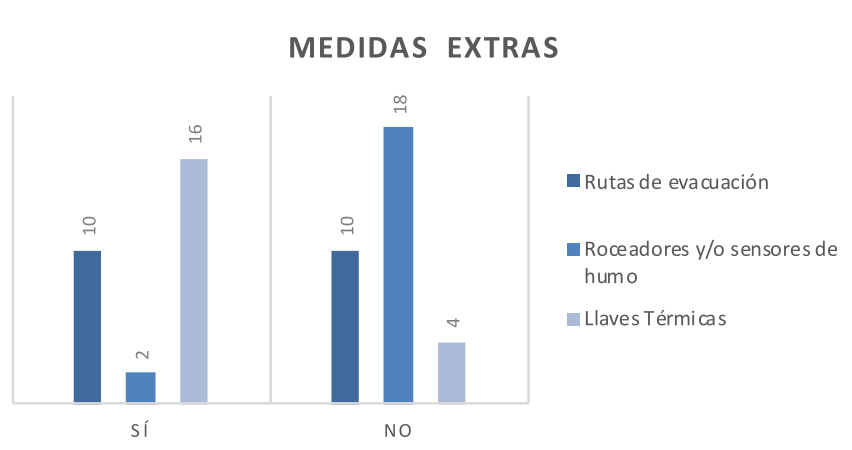

Gráfico 5: Dimensión Generativa de la cultura preventiva en los comerciantes del Mercado Anexo I Piura

En la dimensión generativa se pudo encontrar que 10 de los 20 evaluados cuentan con rutas de evacuación en caso de emergencias dentro de sus puestos de trabajo; sin embargo, los otros 10 manifiestan no tenerlas dentro del local, pero que el mercado en general si cuenta con estas rutas y/o señalizaciones de salida en caso de alguna emergencia. Por otro lado, 18 de los 20 evaluados refieren tener como mínimo con un extintor en la tienda, debido a que la comisión del mercado ACOMIN y Defensa Civil se los exige. Asimismo, 2 de los 20 evaluados refieren tener rociadores de humo y/o sensores de humo dentro de su local, mientras que el resto afirma que el mercado cuenta con sensores de humo, pero carece de rociadores dentro del establecimiento. También, 7 de los 20 evaluados cuenta con botiquín de primeros auxilios. De igual modo, 16 de los 20 evaluados manifiestan tener llaves térmicas de luz como medida de precaución, también conocidas por los encuestados como cuchillas automáticas. Por su parte, 10 de los 20 evaluados refieren que conocen el contacto de los bomberos, el cual se encuentra predeterminado en sus celulares; no obstante, no lo tienen registrado, sino que en caso de emergencia lo encontrarán en sus teléfonos móviles. Finalmente, 1 de los 20 evaluados afirma haber dejado de vender espuma en aerosol como medida de prevención ante incendios; mientras que los otros 19 han mantenido sus mismas ventas y/o actividades, haciendo referencia que dejando de vender perderían sus ingresos habituales. 


\section{DISCUSIÓN Y CONCLUSIONES}

En la investigación se buscó analizar la cultura preventiva frente a incendios urbanos en los comerciantes del Mercado ANEXO I de Piura, a partir de lo cual se encontró que existe una carencia en relación con cultura preventiva, con base en las estadísticas que brinda el cuerpo de bomberos de la ciudad de Piura y los resultados tras aplicada la encuesta. En tal sentido, los comerciantes, antes del incendio, se encontraban en un estado de cultura preventiva en un nivel patológico y, después del siniestro, se ubican en el nivel reactivo. Según Parker, Lawrie \& Hudson (2006), para determinar una cultura preventiva patológica en una empresa, se evidencia que esta actúa en caso de sanción o imposición por parte de la autoridad; entretanto, en el nivel reactivo las empresas actúan cuando el accidente ya se ha producido. A partir de estos supuestos, es posible afirmar que la realidad en dicho establecimiento es que tanto las autoridades, como los comerciantes se encuentran en total indiferencia por prevenir un siniestro, careciendo de una cultura preventiva frente a incendios, por lo que lamentablemente aún existen falencias, tales como mínimamente saber usar un extintor.

En la búsqueda de conocer cómo se presenta la dimensión patológica se encontró que aquellos que habían sido sancionados por no cumplir con el reglamento establecido por Defensa Civil, al levantar las observaciones, ni las autoridades ni los mismos comerciantes previeron que esto podría desatar un desastre. Según Del Prado (2019), las inspecciones resultan importantes porque ayudan a descubrir si cada establecimiento cuenta con equipos desgastados y/o ya han llegado a su límite de condición, ayudando a detectar los métodos y las prácticas que poseen potencialidad de daño; por lo que se trata de un trabajo en conjunto por parte de las autoridades y la comunidad, o en este caso los comerciantes; sin embargo, si organizaciones principales como Defensa Civil son las primeras en realizar su trabajo de manera eficaz y en desconocer parte de sus funciones en la reducción del riesgo y la necesidad de integrar la gestión del riesgo en la planificación de la gestión municipal, es muy probable que ocurran estos grandes accidentes, como plantea Rastelli (2013).

A su vez, con el objetivo de conocer la dimensión reactiva, los resultados arrojaron que los comerciantes se han preocupado por implementar medidas de seguridad contra incendios tras ocurrido el siniestro en el año 2016; esto indica que los encargados de los puestos de trabajo tomaron medidas preventivas después del hecho y tras la ordenanza de Defensa Civil. Esto se explica, como lo plantean las macroempresas, porque los/as trabajadores/as requieren de incentivos o castigo por parte de sus directivos/as para motivarlos a llevar a cabo acciones preventivas (CPS Grupo, 2019); en este caso, ha tenido que ocurrir un incendio de gran magnitud en que se vean afectados para recién acatar la normativa.

Con relación a la dimensión formalista de la variable cultura preventiva en los comerciantes, se encontró que después del siniestro y su reactivación, los comerciantes levantaron observaciones realizadas por ACOMIN y/o Defensa Civil: tienen a la vista su extintor con su respectiva señalización, botiquín, llave térmica de luz en buen estado; sin embargo, estas no se cumplen en un $100 \%$. Estos resultados se contrastan con lo expuesto por Ordoñez (2015), quien concluyó que apenas el $40 \%$ de los establecimientos reúnen todas las normas mínimas requeridas para la prevención de incendio, además, se pudo determinar que los propietarios no tienen conocimiento de los reglamentos y/o normas de prevención ante incendios.

Así mismo, en la dimensión proactiva, los comerciantes en teoría tienen en orden lo que Defensa Civil solicita; aunque, en la práctica es otra realidad, por ejemplo: desconocían el manejo de extintores y el uso de las alarmas de emergencia; aunque contaban con botiquín en su puesto de trabajo, estos no estaban bien implementados con 
los medicamentos de primeros auxilios, inclusive, existía una deficiencia de coordinación entre los mismos trabajadores, ya que no programan capacitaciones y/o simulacros para actuar en conjunto en caso de incendios (ejemplo distribución de roles y funciones). Como afirmó Hernández (2011), usualmente las Mypes ignoran la existencia de un plan de emergencia, nunca se han capacitado respecto a gestión del riesgo, ni participado en simulacros; así mismo, no conocen sobre primeros auxilios e ignoran cómo usar un extintor.

Finalmente, se tuvo como objetivo conocer la dimensión generativa de la variable cultura preventiva en los comerciantes. Al respecto, se encontró que los encargados del Mercado Anexo 1 no llegan a un nivel de cultura generativo, dado que aún no asumen en su totalidad la responsabilidad de prevención, porque se les hace más fácil dejar que otros se hagan cargo, cumpliendo ellos con lo básico para no ser sancionados; así mismo, se percibe desconocimiento con quién acudir y cómo actuar en caso de emergencia o incendio, quedándose sólo en un nivel formalista. Estos resultados se corresponden con lo que Neuhaus (2013) explica con relación a cómo las estrategias pensadas para promover una cultura de prevención y de gestión del riesgo de desastres en el país resultan poco reales, porque no generan compromiso con el tema, aunque esto signifique grandes pérdidas para ellos.

\section{CONSIDERACIONES FINALES}

Se concluye que el incendio ocurrido en el año 2016 en el Mercado Anexo I se produjo por la falta de supervisiones rigurosas sobre las medidas de prevención contra incendios; no obstante, al realizar la inspección solicitada por defensa civil, ciertos comerciantes se dispusieron a levantar las observaciones, pero no se previno que se realizarían actividades de riesgo (ej. soldaduras o cambio de materiales de los puestos) dentro de un lugar altamente inflamable, teniendo como resultado el incendio antes mencionado. Tras ocurrido el siniestro, una parte de los comerciantes han creído conveniente invertir en la instalación de extintores, sensores de humo, rociadores, señalizaciones y alarmas de emergencia, en vez de verse frente a pérdidas de mayor suma con tales accidentes, creando una cultura reactiva en ellos. Sin embargo, como se pudo apreciar, en los trabajadores aún no existe una cultura preventiva adecuada por lo cual es necesario que, en primer lugar, los directivos den a conocer los lineamientos de prevención de manera clara y puntual y, en segundo lugar, elaborar programas formativos de corte teórico-práctico de intervención sobre el manejo de sistemas y/o equipos de protección contra incendios.

\section{RECOMENDACIONES}

Patológica: se recomienda a las autoridades de la comisión ACOMIN programar supervisiones dos veces por año para constatar que los puestos de trabajo cumplan con las normativas, tales como la revisión de cableado, renovación de extintores, llaves térmicas, etc.

Reactiva: informar a los encargados de los puestos las causas de un incendio y las consecuencias que este puede traer.

Formalista: se recomienda que el Cuerpo General de Bomberos instruya al personal sobre el uso adecuado de extintores y/o las entidades supervisoras constanten que el personal reciba las charlas respectivas sobre el uso adecuado del extintor.

Proactiva: informar sobre las medidas de prevención contra incendios que deben tener a los encargados de los puestos de trabajo del Mercado Anexo I.

Generativa: concientizar acerca de la importancia de la seguridad laboral a los encargados de los puestos de trabajo del Mercado Anexo I. 


\section{REFERENCIAS BIBLIOGRÁFICAS}

A un año del incendio, mercado Anexo sigue siendo un riesgo. (23 de noviembre de 2017). Cutivalú. Recuperado en: https://www.radiocutivalu.org/

Alvarado, M. (2015). Evaluación de la gestión de riesgo a los desastres en los procesos administrativos en la educación universitaria superior (tesis para grado de doctor). Universidad de Istmo, Panamá.

El Cuerpo General de Bomberos Voluntarios del Perú (2020). Estadísticas de emergencias a nivel nacional del CGBVP. Perú, Lima. Recuperado en: http://www. bomberosperu.gob.pe/portal/net_estadistica.aspx

Del Canto, E. y Silva, A. (2013). Metodología cuantitativa: abordaje desde la complementariedad en ciencias sociales. Revista de Ciencias Sociales, 3 (141), 25-34.

Del Prado, J. (2019). Funciones del inspector de seguridad y salud ocupacional. IMF Business School Recuperado en: https://blogs.imf-formacion.com/ blog/prevencion-riesgos-laborales/sin-categoria/ inspector-de-seguridad-y-salud/

ENVIRA (2018). ¿Qué es cultura de prevención o cultura preventiva? Recuperado en: https:/envira.es/es/ cultura-de-prevencion-o-cultura-preventiva/

Hernández, J. (2011). Conocimiento y formación en cultura preventiva de riesgo en los Centros Enseñanza Obligatoria de la provincia de Las Palmas y su capacidad de respuesta ante una situación crítica (Tesis doctoral). Universidad de las Palmas de Gran Canaria. España.

Instituto Nacional de Defensa Civil (2010). Incendio urbano. Recuperado en: https://www.munisanta. gob.pe/documentos/incendio_urbano.pdf

Instituto Nacional de Defensa Civil (2013). Incendio Urbano afecta el distrito de Sullana-Piura. Informe de Emergencia $\mathrm{N}^{\circ}$ 003. Recuperado en: https://www.indeci.gob.pe/wp-content/ uploads/2018/12/20130103072433.pdf

Instituto Nacional de Defensa Civil - INDECI (2017). Compendio estadístico del INDECI 2017: Gestión Reactiva. Recuperado en: https://www.indeci.gob.pe/ wp-content/uploads/2019/01/201802271714541.pdf

Instituto Nacional de Defensa Civil (2018). Incendio urbano. Recuperado en: https://www.indeci.gob.pe/ recomendaciones/incendio-urbano/

Mercado Anexo de Piura no tiene un sistema de alerta contra incendios. (5 de febrero de 2018). Cutivalú. Recuperado de: https://www.radiocutivalu.org/ mercado-anexo-piura-no-sistema-alerta-incendios/

Mohammad, N. (2005). Metodología de la investigación ( $2^{\text {a }}$ ed.). México: Limusa. Recuperado en: https:// books.google.com.pe/books?id=ZEJ7-0hmvhwC\& pg=PA91\&dq=dise $\%$ C3\%B1o+descriptivo+simple\& $\mathrm{hl}=\mathrm{es} \& \mathrm{sa}=\mathrm{X} \& v e d=0 \mathrm{ahUKEw} j \mathrm{fysfD} 2 \mathrm{~s} 7 \mathrm{nAhX} 2 \mathrm{GLk}$ GHWlcCNYQ6AEIJzAA\# v=onepage\&q=dise $\% \mathrm{C} 3$ \%B1o\%20descriptivo\%20simple\&f=false

Municipalidad Provincial de Piura (2011). Gerencia de servicios comerciales. Recuperado en: http:// www.munipiura.gob.pe/108-portada/gerencia-deservicios-comerciales

Neuhaus, S. (2013) Identificación de factores que limitan una implementación efectiva de la gestión del riesgo de desastres a nivel local, en distritos seleccionados de la región de Piura (Tesis de maestría). Pontificia Universidad Católica del Perú.

Ordoñez, V. (2015). Estudio sobre el cumplimiento de normas de prevención de incendios de las Pymes en la ciudad de Guayaquil. Universidad Politécnica. Guayaquil, Ecuador.

Parker, D., Lawrie M. \& Hudson, P. (2006). A framework for the development of organizational safety culture. Safety Science. 
Piura: Incendio destruyó 200 puestos del mercado Anexo. (19 de diciembre de 2016). Perú 21. Recuperado en: https://peru21.pe/lima/piura-incendio-destruyo200-puestos-mercado-anexo-fotos-video-236298noticia/?ref=p21r

PREVENCIONAR (2017). La cultura preventiva. Recuperado en: https://prevencionar. com/2017/09/06/cultura-preventiva-3/

Rastelli, V. (2013). La reducción del riesgo ante desastres y la sostenibilidad urbana. Revista Politeia, 53 (37). Instituto de Estudios Políticos, UCV, 2014:81-111

Vázquez, M., Rodríguez, D., Ortíz, N., Olivera, L., Grillo, J. y Bécquer, T. (2017). La prevención del riesgo de desastres en la comunidad. Revista Médica Electrónica, 39(5), $1022-$ 1032. Recuperado de: http://scielo.sld.cu/ scielo.php?script $=$ sci_arttext \&pid $=\mathrm{S} 1684$ $18242017000500002 \& \operatorname{lng}=$ es $\&$ tlng=es.

\section{AUTORES}

\section{Mirian Yanet Velasco Pasapera}

Estudiante de la escuela de psicología, noveno ciclo, de la Universidad César Vallejo, Perú. Correo: mvelascop@ucvvirtual.edu.pe

\section{Jimena Moscol Seminario}

Estudiante de la escuela de psicología, décimo ciclo, de la Universidad César Vallejo, Perú. Correo: jmoscol@ucvvirtual.edu.pe

\section{Lady Diana Celi Zapata}

Estudiante de la escuela de psicología, noveno ciclo, de la Universidad César Vallejo, Perú. Correo: ladyceli64@gmail.com

\section{Sheyla Alexandra Cornejo Medina}

estudiante de la escuela de psicología, onceavo ciclo, de la Universidad César Vallejo, Perú. Correo: pasache4@hotmail.com 